\title{
Advances on pathogenesis of migraine in cold regions
}

\author{
Qihui Chen ${ }^{1}$, Yonghui Pan ${ }^{1 *}$, Dan Yao ${ }^{1}$, Yongchen Wang ${ }^{2^{*}}$
}

\begin{abstract}
Migraine is a common primary headache which seriously affects the quality of patients' life due to the high prevalence and disability rate. Recent years a large number of studies have found that temperature is directly bound to migraine and migraine patients in cold regions have higher prevalence, different manifestations and poor response to the conventional therapy. We propose in this review article a new concept of migraine in cold regions on the basis of geography and summarize the research advances on the pathogenesis of migraine in cold regions to provide conceptual basis for the clinical diagnosis and treatment of this disease entity.
\end{abstract}

\section{Keywords}

migraine; cold regions; pathogenesis; genetics

Received 26 June 2021, accepted 2 November 2021
${ }^{1}$ Department of Neurology, the First Affiliated Hospital of Harbin Medical University, Harbin 150001, China

${ }^{2}$ The Second Affiliated Hospital of Harbin Medical University, Harbin 150001, China

*Corresponding author Yonghui Pan, E-mail: aigui1993@126.com; Yongchen Wang, E-mail: yongchenwang@163.com

\section{Understanding of migraine in cold regions}

Migraineis a common neurobiological headache disorder, characterized by unilateral headache from moderate to severe intensity. It is estimated that migraine may affect over 1 billion people worldwide and the 1-year period prevalence in China is about $9 \%{ }^{[1]}$. According to the recurrent headache, higher disease cost and prevalence of mental complications, migraine has been listed as one of the most disabling diseases by the World Health Organization (WHO) in $2001^{[2]}$, directly and indirectly increasing the medical burden on both individuals and society.

Multiple triggerring factors of migraine have been identified through clinical trials which can be roughly divided into internal causes such as genetic susceptibility, endocrine disorder, stress and sleep disturbances and external causes like environment, chemicals, certain food and drugs. A study with statistical analysis on the triggers of the acute migraine attack in 1207 patients revealed that stress, hormones (female), fasting, and weather changes were the most common factors ${ }^{[3]}$. Notably, in cold environement, the incidence of migraine is significantly increased and patients usually present higher headache intensity ${ }^{[4-5]}$, suggesting low temperature may have an effect on the pathophysiological process of migraine. According to the definition by geographic scholars, cold regions refer to the areas with average temperature of the coldest month is $<-3^{\circ} \mathrm{C}$, months over $10^{\circ} \mathrm{C}$ $\leq 5$, and annual average temperature $\leq 5^{\circ} \mathrm{C}$ and are mainly located in the Heilongjiang province, the east and north of Jilin province and the northeastern of Inner Mongolia autonomous region ${ }^{[6]}$. We therefore propose here a concept of "Migraine in Cold Regions" on the basis of geographical distribution, which can be distinguished from the migraine in common areas. Specifically, migraine in cold regions is accompanied by higher prevalence, special clinical manifestations and unique pathogenesis. Migraine in cold regions should be regarded as an independent subtype which is worthy of a further studying on its prevalence, pathogenesis and clinical features.

\section{Research status of migraine in cold regions}

A study collected 4700 migraine-relevant messages regarding the relationship between frequency of migraine attacks and temperature changes through smartphone softwares and web forms, and found that every $5^{\circ} \mathrm{C}$ temperature drop increased the messages of migraine by about $20 \%{ }^{[4]}$.

Besides higher prevalence, the clinical manifestations of migraine in cold regions also have other uniqueness. One study reported that the frequency of migraine attacks in cold weather increases with increasing ages ${ }^{[7]}$. Hoffmann et al. ${ }^{[5]}$ retrospectively evaluated the clinical data of 20 migraine patients ( 5 males and 15 females) in one year period (January to December, for excluding seasonal deviation) and recorded 
frequency, location, duration, character and intensity of headache and the related weather data. Their results showed that low temperature was linked to the frequency and intensity of migraine with daily headache attacks occurring most frequently and obviously at 4 am and monthly events peaking in January and bottoming in August. Additionally, a large scale study of 4 821 migraine patients ( 1059 males and 3762 females) in China found temperature changes increase the incidence of aura symptoms such as neck stiffness, dizziness, yawn and lethargy ${ }^{[8]}$. Based on this, it is further speculated that most migraine patients in cold regions may suffer from aura symptoms, which may be caused by the cortical spreading depression (CSD). In summary, the prevalence, attack frequency and clinical manifestations of migraine in cold regions possess different characteristics from those of migraine in common regions.

There are no widely accepted defnition and diagnostic criteria of migraine in cold regions. At present, the characteristics are mostly deduced indirectly from relevant studies without definite data supporting. Thus epidemiological investigations and analysis of clinical manifestations on migraine in cold regions are of paramount importance for improving our understadning of migraine in cold regions.

\section{Possible pathogenesis of migraine in cold regions}

A large number of studies have shown that cold stress can cause abnormalities in blood flow, constriction and dilation of cerebral vessels, ion channel function, hyperexcitability or inhibition of neurons, and on the like ${ }^{[9]}$. Although the mechanism of low temperature-induced migraine is unclear, genetics may play a crucial part by setting the genetic suceptibility to the pathogenesis of migraine in cold regions. A number of hypotheses on the pathogenesis of migraine in cold regions have been proposed by the basis of migraine's pathogenesis and low temperature-induced pathological process.

\subsection{Transient receptor potential channel hypothesis}

Transient receptor potential (TRP) channels are a large class of non-selective cation channels which are expressed on cytoplasmic membrane and organelle membrane. According to their amino acid sequence homology, TRP channels are mainly divided into six subfamilies including transient receptor potential melastatin (TRPM), transient receptor potential vanilloid (TRPV), transient receptor potential ankyrin (TRPA), etc ${ }^{[10]}$. Functionally, they serve as the sensors of temperature and noxious stimuli and the activation mechanism of meningeal nociceptors to participate in the process of inflammatory pain ${ }^{[11]}$, which is an important pathway related to the cold-induced migraine attacks.

\subsubsection{TRPM8 channel}

TRPM 8 is an ion channel sensitive to low temperature $\left(<26^{\circ} \mathrm{C}\right.$ ), pressure, and cooling compounds (such as menthol and nicholin) and is abundantly expressed in neural circuits related to migraine pathogenesis including dorsal root ganglion and trigeminal ganglion ${ }^{[12]}$. A study found that the pain behavior caused by specific activation of TRPM8 in dura mater was consistent with migraine and the anti-migraine agent sumatriptan significantly relieved the hyperalgesia caused by TRPM8 agonist nicholin on epidural, suggesting that the activation of TRPM8 in dura mater is involved in the occurrence of migraine ${ }^{[13]}$. It is believed that the pathogenesis of migraine is associated with calcitonin gene related peptide (CGRP). A recent animal study demonstrated that ablation of CGRP- $\alpha$ in sensory neurons enhanced the TRPM8-mediated behavioral response to cold stimulation, and functionally interacts with TRPM8 to promote migraine attacks ${ }^{[14]}$. Meanwhile, TRPM8 has also received attention as a drug target; the analgesic mechanism of TRPM8 is mainly attributed to its action on group II/III metabotropic glutamate receptors (mGluR) through central-mediating to inhibit nociceptive inputing ${ }^{[15]}$. Currently, the development of TRPM8-targeting drugs has demonstrated promising prospect presenting a new direction for the treatment of migraine.

A Spain study in 2016 conducted a case-control study on 12 single nucleotide polymorphism (SNP) in 512 migraine patients with aura symptoms and 535 healthy controls found that rs12134493 locus of TRPM8 gene was associated with migraine ${ }^{[16]}$, and this result was further verified in migraine without aura symptoms of Han population in Southwest China ${ }^{[17]}$. Genovariation of TRPM8 leads to the substitution of amino acid and affects the expression level and function of calcium ion channels. TRPM8 genes have been consistently identified as susceptive genes for migraine in many genome-wide association studies from various countries.

Another study identified the association of six pre-identified migraine susceptibility SNPs with migraine subtypes and endophenotypes, including chronic migraine and allodynia ${ }^{[18]}$. Of particular note, among the six SNPs, TRPM8 rs10166942 $\mathrm{T}$ allele-carrying patients were more likely to develop into chronic migraine, a more severe type of migraine than non- $T$ allele carriers. In addition, T allele carriers have more allodynic symptoms than non-T allele carriers ${ }^{[18]}$, suggesting that migraine patients with TRPM8 gene mutation is associated with more serious clinical manifestations.

A large-scale female-based study which included 3003 migraine 
patients and 18108 normal controls unraveled that rs7577262/ TRPM8 was associated with migraine, but showed little selectivity to any characteristics of migraine ${ }^{[19]}$. However, a rs7577262/TRPM8 correlation study conducted in Fujian province of China obtained an opposite result ${ }^{[20]}$. Fujian province is located in the southeast coastal area of China and has a typical subtropical climate with an annual average temperature of about $26^{\circ} \mathrm{C}$. The descrepancy reflects that the expression of TRPM8 gene, as a cold stimulation sensor, depends on the climate. Migraine patients in cold regions may be accompanied with special genetic mutations, resulting a higher headache attack frequency and more severe headache attack form. Yet more rigorous studies are needed to clarify this notion.

\subsubsection{TRPA1 channel}

TRPA1 is primarily located in the subpopulation of primary sensory neurons of trigeminal, vagal and dorsal root ganglia and it can be activated by cold stimulation, harmful chemicals and a number of exogenous compounds (including molecules of botanical origin and environmental irritants). Upon activation of nociceptors, the neurons can produce and release the neuropeptides substance $P(S P)$ and CGRP to induce migraine attacks through neurogenic inflammatory responses. In the same time, the TRPA1-oxidative stress system is the primary transducing pathway producing pain and neurogenic inflammatio and it plays a vital role in the transition of mechanical and cold hypersensitivity from an acute to a chronic condition ${ }^{[21]}$. The drugs targeting TRPA1 elicit their effects by inhibiting release of CGRP from trigeminal neurons through neuronal desensitization and the meningeal vasodilation mediated by CGRP ${ }^{[22]}$.

The human TRPA1 gene is located on q21.11 band of chromosome 8 and contains 29 exons. A study analyzed the variants in 170 migraine patients and 173 controls through genotyping and revealed that c.3053A>G TRPA1 (pH1018R, rs959976) polymorphism was more common in patients with migraine onset before the age of 15 , though no statistical differences were found in the frequency of c.3053A>G TRPA1 between the migraine and control groups ${ }^{[23]}$. Although no specific mutation of TRPA1 genes was found in migraine patients, rs1198795 (TRPA1) was related to pain response caused by low temperature. Comparing with G/G homozygous patients, patients carrying A/A homozygous variants have significantly shorter cold withdrawal time and lower pain tolerance to cold stimulation ${ }^{[24]}$. The existing research results suggest a possibility of certain mutations related to cold stimulation in TRPA1 genes of migraine patients in cold regions, but future studies are required to verify this point.

\subsubsection{TRPV1 channel}

TRPV1 is mainly distributed in small neurons in dorsal root ganglion and trigeminal ganglion of spinal cord, participating in sensing pain caused by temperature and chemical stimulation. However, compared with cold stimulation, TRPV1 is more sensitive to thermal stimulation (> $\left.42{ }^{\circ} \mathrm{C}\right)^{[25]}$. Studies have shown that TRPV1 immunoreactive peripheral blood vessels and stromal nerve fibers are densely distributed in the meninges, and some TRPV1 immunoreactive nerve fibers are co-expressed with proteinaseactivated receptor-2 (PAR2) ${ }^{[26-27]}$, which is linked to the pain induced by tryptase release by meningeal mast cells activating PAR2.

Recently, a study found that the expression of TRPV1 mRNA and protein was significantly up-regulated in the dorsal spinal cord tissues in a rat model of chronic constriction injury ${ }^{[28]}$. TRPV1 up-regulation apparently increased mechanical allodynia and thermal hyperalgesia as well as the expression of inflammationassociated genes (COX-2, TNF- $\alpha$, and IL-6). Moreover, TRPV1 was identified as a downstream target of miR-338-3p and overexpression of TRPV1 restrained the inhibitory effect of miR338-3p on neuropathic. It appears that TRPV1 has the potential to be a target for the treatment of neuropathic ${ }^{[28]}$. Clearly, further studies on this pathway may yield desirable results for the development of novel therapeutic strategy of migraine, a typical neuroinflammatory disease.

\subsection{Changes in cold sensitivity}

A study conducted in China analyzed 66 migraine patients (75.8\% female, and the average age was $43.3 \pm 12.9$ years old) by monitoring the symptoms of headache and temperature changes within one year. The results exihibit that the relative contribution to headache atributable to temperature changes is $16.5 \%$ in winter and $9.6 \%$ in summer, and temperature changes can explain $27 \%$ of mild headache attack in winter ${ }^{[29]}$. Individuals who are sensitive to temperature are more likely to have headache aggravation in winter, especially in patients with mild headache, suggesting that migraine patients are more sensitive to cold stress. The genetic factors may as a determinant of higher sensitivity to weather flutuations ${ }^{[29]}$, many studies have confirmed that the upregulation of TRPM8 expression is one of the important causes for the increase of cold sensitivity, which is mainly related to the calcium-independent phospholipase A2 (iPLA2) pathway. Additionally, cold and menthol stimulation can lead to calcium release from endoplasmic reticulum, activation of iPLA2 following calcium depletion from intracellular pools, release of lysophosphatidylcholine (LPC) via the iPLA2 pathway. These alterations confer cold hypersensitivity in a TRPM8dependent manner. Consistently, inhibition of the iPLA2 pathway eliminates the TRPM8-provoked response to cold. Furthermore, 
the activation of TRPM8 induced by cold and menthol requires the presence of membrane phosphatidylinositol-4,5-bisphosphate (PIP2), and PIP2 by itself can also activate this channel ${ }^{[30]}$. Collectively, available research results uncovered the cause of higher prevalence of migraine in cold regions and also suggest that genetic research on TRPM8 be a key direction in the future.

\subsection{Other possible mechanisms}

Weather changes is not only limited to the changes of temperature, they also include changes in humidity, air pressure, wind speed and on the like. These alterations interact among one another and fluctuate dynamically along the time. A recent study indicates that higher humidity is associated with higher probability of migraine in warm season and traffic-related gaseous pollutants (such as ozone and carbon monoxide) may be related to higher probability of migraine in cold season $^{[31]}$, indicating that the influence of temperature on migraine is a complex process of multifactorial interactions and temperature changes may also aggravate migraine by affecting other factors. On the other hand, due to the influence of geographical environment, the economic level in cold regions is relatively backward and the disease treatment approaches and the accessibility of patients to health/medcincine-related facilities is relatively limited compared to other regions, which can impose negative impacts on the prevalence, severity and prognosis of migraine.

\section{Summary and prospect}

In this article, we put forward the concept of "Migraine in Cold Regions" and provided an overview of the research status related to this concept. Migraine in cold regions is accompanied by higher prevalence and attack frequency and more serious clinical manifestations, and its impact on patients' quality of life is much higher than migraine in common regions. It should be noted that the research on migraine in cold regions is challenged by many difficulties. First, the impact of environmental parameters on the health status is usually concealed and progressive, and a large number of patient samples and a longer time span are required to obtain statistically significant and pathophysiologically meaningful results. At the same time, univariate analysis on the variable temperature may not yield quantitatively precise results and it is hard, if not impossible, to accurately control the variables. As a result, the current research results on the relationship between migraine and temperature are less consistent and even conradictory. Therefore, how to study the incidence, clinical characteristics and genetics of migraine in cold regions through rigorous experiments is an important issue to be solved and also a focus in the future research.

\section{Conflicts of interests}

Yongchen Wang is an Editorial Board Member of the journal. The article was subject to the journal's standard procedures, with peer review handled independently of this member and his research groups.

\section{Acknowledgements}

This project was supported by General Program of National Natural Science Foundation of China (82071549); Key Program of Natural Science Foundation of Heilongjiang Province (ZD2019H006); Key Program of Planning Subject for the 13th Five-Year Plan of Heilongjiang Province Education Sciences (GJB1319086).

\section{References}

[1] Ashina M, Katsarava Z, Do T P, et al. Migraine: epidemiology and systems of care. Lancet, 2021; 397(Suppl 3): S0140-6736(20)32160-7.

[2] Jensen R, Stovner L J. Epidemiology and comorbidity of headache. Lancet Neurol, 2008; 7(4): 354-361.

[3] Kelman L. The triggers or precipitants of the acute migraine attack. Cephalalgia, 2007; 27(5): 394-402.

[4] Scheidt J, Koppe C, Rill S, et al. Influence of temperature changes on migraine occurrence in Germany. Int J Biometeorol, 2013; 57(4): 649-654

[5] Hoffmann J, Lo H, Neeb L, et al. Weather sensitivity in migraineurs. J Neurol, 2011; 258(4): 596-602.

[6] Chen R, Kang E, Wu L, et al. Cold regions in China. Journal of Glaciology and Geocryology, 2005; 27(4): 469-475.

[7] Akgün N, Acıman Demirel E, Açıkgöz M, et al. The effect of weather variables on the severity, duration, and frequency of headache attacks in the cases of episodic migraine and episodic tension-type headache. Turk J Med Sci, 2021; 51(3): 1406-1412.
[8] Wang X L, Yin Z M, Lian Y J, et al. Premonitory symptoms in migraine from China: a multi-clinic study of 4821 patients. Cephalalgia, 2021; 41(9): 991-1003.

[9] Vgontzas A, Burch R. Episodic migraine with and without aura: key differences and implications for pathophysiology, management, and assessing risks. Curr Pain Headache Rep, 2018; 22(12): 78.

[10] Hung C Y, Tan C H. TRP channels in nociception and pathological pain. Adv Exp Med Biol, 2018; 1099: 13-27.

[11] Benemei S, Dussor G. TRP channels and migraine: recent developments and new therapeutic opportunities. Pharmaceuticals (Basel), 2019; 12(2): 54

[12] Dussor G, Cao Y Q. TRPM8 and migraine. Headache, 2016; 56(9): 1406-1417.

[13] Burgos-Vega C C, Ahn D D, Bischoff C, et al. Meningeal transient receptor potential channel M8 activation causes cutaneous facial and hindpaw allodynia in a preclinical rodent model of headache. 
Cephalalgia, 2016; 36(2): 185-193

[14] McCoy E S, Zylka M J. Enhanced behavioral responses to cold stimuli following CGRPa sensory neuron ablation are dependent on TRPM8. Mol Pain, 2014; 10: 69.

[15] Proudfoot C J, Garry E M, Cottrell D F, et al. Analgesia mediated by the TRPM8 cold receptor in chronic neuropathic pain. Curr Biol, 2006; 16(16): 1591-1605

[16] Sintas C, Fernández-Morales J, Vila-Pueyo M, et al. Replication study of previous migraine genome-wide association study findings in a Spanish sample of migraine with aura. Cephalalgia, 2015; 35(9): 776782.

[17] Wang $P$. The research on the correlation between gene polymorphism such as PRDM16, TRPM8, TSPAN2, MMP16etc and migraine without aura. Sichuan Med J, 2016; 37(2): 131-134. (in Chinese)

[18] Ling Y H, Chen S P, Fann C S, et al. TRPM8 genetic variant is associated with chronic migraine and allodynia. J Headache Pain, 2019; 20(1): 115.

[19] Chasman D I, Anttila V, Buring J E, et al. Selectivity in genetic association with sub-classified migraine in women. PLoS Genet, 2014; 10(5): e1004366

[20] Fu X, Yang J, Wu X, et al. Association between PRDM16, MEF2D, TRPM8, LRP1 gene polymorphisms and migraine susceptibility in the She ethnic population in China. Clin Invest Med, 2019; 42(1): E21-E30. [21] Nassini R, Materazzi S, Benemei S, et al. The TRPA1 channel in inflammatory and neuropathic pain and migraine. Rev Physiol Biochem Pharmacol, 2014; 167: 1-43.

[22] Materazzi S, Benemei S, Fusi C, et al. Parthenolide inhibits nociception and neurogenic vasodilatation in the trigeminovascular system by targeting the TRPA1 channel. Pain, 2013; 154(12): 27502758.

[23] Kowalska M, Prendecki M, Kapelusiak-Pielok M, et al. Analysis of genetic variants in SCN1A, SCN2A, KCNK18, TRPA1 and STX1A as a possible marker of migraine. Curr Genomics, 2020; 21(3): 224-236.

[24] Naert R, Talavera A, Startek J B, et al. TRPA1 gene variants hurting our feelings. Pflugers Arch. 2020; 472(7): 953-960.

[25] Bevan S, Quallo T, Andersson D A. TRPV1. Handb Exp Pharmacol, 2014; 222: 207-245

[26] Dux M, Rosta J, Messlinger K. TRP channels in the focus of trigeminal nociceptor sensitization contributing to primary headaches. Int J Mol Sci, 21(1): 342.

[27] Zhang X C, Levy D. Modulation of meningeal nociceptors mechanosensitivity by peripheral proteinase-activated receptor-2: the role of mast cells. Cephalalgia, 2008; 28(3): 276-284.

[28] Ma Y T, Deng Q L, Li S G, et al. TRPV1, Targeted by miR-338-3p, induces neuropathic pain by interacting with NECAB2. J Mol Neurosci, 2021; 71(1): 55-65.

[29] Horne D B, Biswas K, Brown J, et al. Discovery of TRPM8 antagonist (S)-6-(((3-Fluoro-4-(trifluoromethoxy)phenyl)(3-fluoropyridin2-yl)methyl)carbamoyl) nicotinic Acid (AMG 333), a clinical candidate for the treatment of migraine. J Med Chem, 2018; 61(18): 8186-8201.

[30] Liu Y, Mikrani R, He Y, et al. TRPM8 channels: a review of distribution and clinical role. Eur J Pharmacol, 2020; 882: 173312.

[31] Li W, Bertisch S M, Mostofsky E, et al. Weather, ambient air pollution, and risk of migraine headache onset among patients with migraine. Environ Int, 2019; 132: 105100. 